\title{
Dynamics of small molecules within the F127 PEO-PPO-PEO triblock copolymer gel and sol phases studied at the molecular scale
}

\author{
Suma S. Thomas ${ }^{1,2}$, Helia Hosseini-Nejad ${ }^{1,2}$ and Cornelia Bohne ${ }^{1,2 *}$ \\ ${ }^{1}$ Department of Chemistry, University of Victoria, PO Box 1700 STN CSC, Victoria, BC V8W 2Y2, Canada \\ ${ }^{2}$ Centre for Advanced Materials and Related Technology (CAMTEC), University of Victoria, 3800 Finnerty Rd, \\ Victoria, BC, V8P 5C2, Canada
}

\begin{abstract}
Triplet excited states of guest molecules with different hydrophobicities were used to probe the association and dissociation dynamics of these guests with F127 micelles in the gel and sol phases. The dynamics probed was on a longer length scale than amenable with fluorescence techniques, but at a shorter length scale than probed in translational diffusion studies. The mobility of the guests at the molecular scale showed that subtle changes in the guest's structure affect the guest's release time from the micelles, where the structural features of the guest are more important than the phase, gel vs. sol, of the system.
\end{abstract}

\section{Introduction}

Hydrogels are viscoelastic materials with structured and confined environments. These materials can act as barriers between liquid or gaseous interfaces, and can adhere to compatible surfaces. Hydrogels have found applications in varied fields, such as oil exploration, ${ }^{1-3}$ food and cosmetics industries, ${ }^{4-6}$ and as specialized functional materials for drug delivery and in bioengineering. ${ }^{6-11}$ For some applications, control of small-molecule mobility within the gel or to the surrounding environment is key to achieving the intended function of the material. Knowledge on the effect of the hydrogel's structure on the internal mobility of small molecules will enable the control of this mobility aiding in the rational design of functional gels.

Mobility in hydrogels of molecules spanning different length scales, which corresponds to the molecule's mobility within different volumes, have been studied using a handful of spectroscopic techniques, such as pulsed field gradient nuclear magnetic resonance (PFGNMR), ${ }^{12-14}$ fluorescence recovery after photobleaching (FRAP), ${ }^{15-17}$ fluorescence correlation spectroscopy (FCS), ${ }^{18-22}$ and time-resolved fluorescence anisotropy measurements. ${ }^{16,21,23-25}$ The time resolution for each technique dictates the length scale for which mobility is probed. Fluorescence anisotropy decays occur over nanoseconds, providing information about the guest's local mobilities and microviscosities. FCS is used to measure events from nanoseconds to seconds for a small detection volume with dilute fluorophore solutions. PFGNMR and FRAP track diffusion events that are longer than milliseconds leading to information on average mobilities between different environments in the hydrogel.

A technique with microsecond resolution is required to obtain mobility information for length scales beyond the local environment but not averaged over different environments. Triplet excited states, which have longer lifetimes than the fluorescent singlet excited states, are suitable guests for such studies. Laser flash photolysis (LFP) is used to measure the decay kinetics of triplet excited states. We used LFP to study

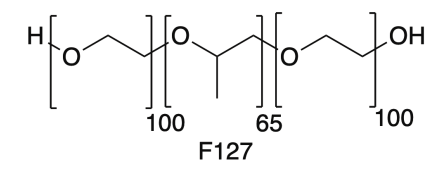

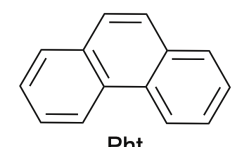

Pht

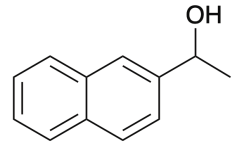

$2-\mathrm{NpOH}$

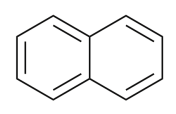

$\mathrm{Np}$

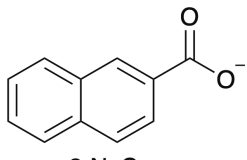

2-NpC
Chart 1. Chemical structures of Pluronic ${ }^{\circledR}$ F127, phenanthrene $(\mathrm{Pht})$, naphthalene $(\mathrm{Np}),(\mathrm{R})-1-(2-$ naphthyl)ethanol $(2-\mathrm{NpOH})$ and 2-naphthoic acid (2-NpC)

the mobility of guests in hydrogels formed from polyethyleneoxide-polypropyleneoxide-polyethyleneoxide (PEO-PPO-PEO, Pluronics ${ }^{\circledR}$ ) triblock copolymers (Chart 1), where guests are located in different binding sites of the Pluronics micelles.

Pluronics are used in applications, such as cosmetics, ${ }^{26-28}$ drug delivery and tissue engineering, ${ }^{7,29-31}$ where the mobility of small molecules within the gel and into a surrounding medium is central to the intended function of these materials. Pluronics form micelles in solution above the critical micelle concentration with a PPO core and a PEO corona. ${ }^{32,}{ }^{33}$ At increased temperatures, the dehydration of the PPO core combined with the entanglement of the corona leads to gel formation. ${ }^{32-34}$ At temperatures around $20-30{ }^{\circ} \mathrm{C}$ hard gels with a crystalline structure are formed, whereas at higher temperatures $\left(>50^{\circ} \mathrm{C}\right)$ dehydration of the PEO corona leads to the collapse of the crystalline structure and the formation of soft gels. ${ }^{35}$ In the sol or gel phases, guest molecules can be located in the PPO micellar core, the PEO corona, the peripheral area of the PEO corona, or the aqueous phase. ${ }^{23,36-}$ 
Previous studies on the mobility of small molecules in Pluronics micelles in solution or in gels led to the determination of diffusion constants and local viscosities, ${ }^{20,23,}$ ${ }^{36-40}$ but no information is available on the mobility of guests into and out of specific binding sites of the micelles. PFGNMR, FRAP and FCS led to the determination of average values for the random translational motions of guest molecules between different environments of the sol and gel systems. For example, FCS has shown that human serum albumin diffuses 59 times slower in the Pluronic F127 (Chart 1) gel compared to the diffusion in water. ${ }^{20}$ This protein experiences a greater resistance while moving in the gel, likely due to the higher viscosity of these gels compared to water. Electron spin resonance measurements showed that a guest tightly bound to the L64 Pluronic system diffuses slowly when compared to a hydrophilic guest. ${ }^{40}$ Fluorescence anisotropy experiments showed that the microviscosity experienced by guests located in different environments of the Pluronic micelles in the sol or gel phase are different. In the gel, these viscosities are much lower than the gel's macroscopic viscosity, ${ }^{23,36,38,39}$ suggesting that the rotational mobility of the guests is determined by the local molecular environment and not the macroscopic viscoelastic properties of the gel. Guests experience a microviscosity gradient with higher values observed for guests in the micellar PPO core and decreasing microviscosities as the guest in the corona is located farther away from the core. Complementary steady-state and time-resolved fluorescence measurements, including excited state quenching studies, were employed to characterize the properties of Pluronic micelles, such as the determination of the polarity of the guest binding site, the aggregation number of the micelles and the accessibility of aqueous quenchers to singlet excited states bound to the micelles. ${ }^{41-43}$ However, the singlet excited state quenching experiments did not yield information on the association and dissociation processes of the guest from the micellar binding sites.

The objective of our study was to determine if the release time of guests from F127 micelles is dependent on the binding site for the guest. The release time is related to the dissociation rate constant of the guest from the micelle. This dissociation process occurs at a molecular scale and is different from the translational diffusion that occurs over longer distances and multiple binding environments or the overall release of a guest from within the gel to an external medium. Based on previous studies with coumarin dyes, ${ }^{23}, 37,38$ we chose polyaromatic guests with different hydrophobicities (Chart 1). These molecules are inherently hydrophobic as indicated by their high partition coefficients in the octanol-water binary solvent system and their hydrophobicity increases with increasing number of aromatic rings in the molecule. ${ }^{44}$ Among the chosen guests, phenanthrene (Pht) is the most hydrophobic followed by naphthalene (Np). R-1-(2-naphthyl)ethanol (2-NpOH) is less hydrophobic than $\mathrm{Np}$ because of the hydrogen bonding ability of the alcohol moiety and 2-naphthoic acid (2-NpC) is the least hydrophobic guest because at the $\mathrm{pH}$ of $6.5 \pm 0.1$ used, $2-\mathrm{NpC}$ $\left(p K_{a}=4.16\right)^{44}$ is deprotonated and is present as the carboxylate anion. These guests readily form triplet excited states and the decay of these excited states was measured using LFP. We used quenching studies, previously used only in solutions, ${ }^{45-50}$ to determine the association and dissociation rate constants of the guests from the F127 micelles in the gel and sol phases. Our studies showed that the binding location in the micelle affects the dissociation rate constant from the micelle. The binding dynamics of the guest in solution and in the gel are similar, but differences were observed in the gel for the guest bound to the corona close to the core compared to the guest located at the periphery of the micelle. This nuanced molecular picture provides predictions on how the structure of guests can be modified to affect the binding dynamics of these guests. This local mobility ultimately will affect the guest's overall mobility in the gel and from the gel to a surrounding medium, providing a pathway on how to design molecules or gels where the release time of small molecules from functional gel materials can be tuned.

\section{Theoretical Concepts}

The theoretical background for the quenching methodology of triplet excited states in compartmentalized systems is provided with a focus on the differences compared to fluorescence studies. The absorption of a photon by a molecule takes it to an excited state, which has a finite lifetime. Consequently, the excited molecule can only move within a defined volume while in its excited state. Triplet excited states have lifetimes in the micro- to millisecond time scales, whereas singlet excited states of most organic molecules return to their ground state with lifetimes of less than tenths of nanoseconds. ${ }^{51}$ Thus, a molecule in its singlet excited state can be treated as stationary during its lifetime, while a molecule in its triplet excited state can move within the system before decaying to its ground state. In a compartmentalized system, like micelles, the triplet excited states can move between different compartments, that is the micellar and aqueous phases, before deactivation occurs. Singlet excited states are emissive, resulting in fluorescence and their lifetimes are determined using time-resolved fluorescence studies. However, the decay of triplet excited states that results in phosphorescence is rare and mostly these excited states undergo non-radiative transitions to their ground state. ${ }^{51}$ As a result in LFP, the triplet excited state decay kinetics is followed by changes in absorption of the triplet excited state and corresponds to a mono-exponential decay yielding an observed rate constant $\left(k_{o b s}\right)$. By definition, $k_{\text {obs }}$ is equal to the reciprocal of the triplet excited state lifetime.

The encounter of an excited state molecule with a quencher (Q) decreases the emission intensity and shortens the excited 


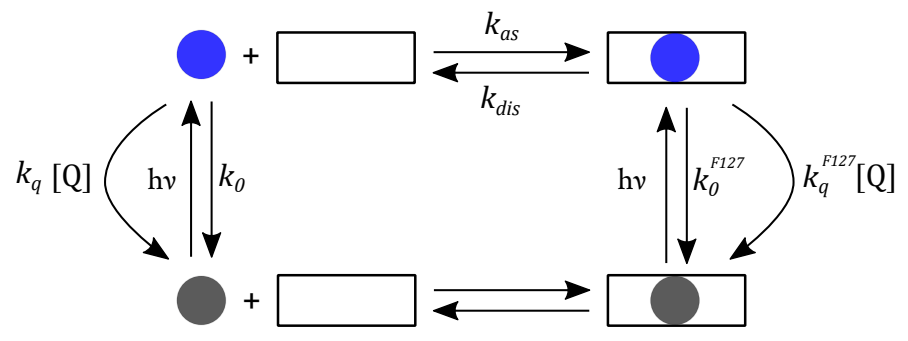

Scheme 1. Kinetic pathways for the excited guest in different compartments. The square indicates the micelle, while the circle is the guest in its ground (grey) or excited state (blue).

state lifetime when quenching requires the diffusion of quencher and excited state to form an encounter complex. ${ }^{52}$ This dynamic quenching leads to a linear relationship between $k_{\text {obs }}$ and quencher concentration, as shown in eqn 1 , where $k_{0}$ is the decay rate constant in the absence of quencher and $k_{\mathrm{q}}$ is the quenching rate constant, which is a measure of the diffusion of the quencher. ${ }^{53} \mathrm{~A}$ different quenching mechanism, static quenching, occurs when the excited state and quencher are located in close proximity and do not diffuse for quenching to occur. Static quenching does not provide kinetic information. ${ }^{52}$ Therefore, dynamic quenching is required to obtain information on the mobility of the excited state or quencher. Dynamic quenching of singlet excited states leads to information on the mobility of quenchers because of the short lifetime of the singlet excited states, ${ }^{54}$ while the mobility of guests is measured by studying the kinetics for triplet excited states. ${ }^{45,53}$

$k_{o b s}=k_{0}+k_{q}[\mathrm{Q}]$

In the present study, the quencher for the triplet excited states of Pht, Np, 2-NpOH and 2-NpC was sodium nitrite $\left(\mathrm{NaNO}_{2}\right)$, where the negatively charged nitrite ion is responsible for the quenching of the excited guest. ${ }^{49}$ This quencher being hydrophilic, resides mainly in the water phase of the F127 sol or gel. In the compartmentalized F127 micellar system, the guest can be located in the micelle or the aqueous phase (Scheme 1). ${ }^{45}$ The quenching by nitrite anion is less efficient for the micellar bound excited guest compared to quenching in the water compartments due to the protection offered by the micelle for the approach of the hydrophilic quencher into the micelle where the excited guest is located. This differential quenching leads to a lower quenching rate constant in the micelle $\left(k_{q}^{F 127}\right)$ compared to that in water $\left(k_{q}\right)$. The ratio between $k_{q}$ and $k_{q}^{F 127}$ corresponds to the protection efficiency (PE) that is related to the extent by which the micelle protects the guest from interactions with the quencher.

The decay kinetics for the triplet excited state is related to the coupling of the quenching rates constants and the guest's association $\left(k_{a s}\right)$ and dissociation rate constants $\left(k_{d i s}\right)$ with the micelle (eqn 2 , where $N$ is the aggregation number for the micelles). The analysis of the curved quenching plot leads to the determination of $k_{a s}$ and $k_{\text {dis. }}$. At high quencher concentrations, the last term in eqn 2 is negligible and the $k_{o b s}$ dependence on the quencher concentration is linear, where the slope corresponds to $k_{q}^{F 127}$.

$k_{o b s}=k_{0}^{F 127}+k_{d i s}+k_{q}^{F 127}[\mathrm{Q}]-\frac{k_{d i s} k_{a s} \frac{[\mathrm{F} 127]}{N}}{k_{a s} \frac{[\mathrm{F} 127]}{N}+k_{0}+k_{q}[\mathrm{Q}]}$

The relevant assumptions used in the derivation of eqn 2 are: (i) The concentration of the triplet excited state guests in the micelles is much higher compared to that in water. This condition allows for the steady-state approximation to be used for the guest concentration in water, leading to an expression for the reaction rate that corresponds to a mono-exponential decay for the triplet excited state. ${ }^{47}$ If a significant fraction of the guest in located in the micelles and in water, then the decay does not follow a mono-exponential function. (ii) Low concentrations of guests are used so that any self-quenching or triplet-triplet annihilation of the excited state molecule is eliminated. ${ }^{47}$ In order to meet the requirement imposed by the second assumption, sufficiently low guest concentrations were used to eliminate the possibility of triplet-triplet annihilation or self-quenching occurring within one micelle containing more than one guest (Table S1).

\section{Experimental}

Materials: Pluronic ${ }^{\circledR} \mathrm{F} 127$ (Sigma-Aldrich, MW 12,600 Da, 70 wt\% PEO), naphthalene (Np, Sigma-Aldrich, $\geq 99 \%)$, phenanthrene (Pht, Sigma-Aldrich, $\geq 99.5 \%)$, (R)-1-(2naphthyl)ethanol (2-NpOH, Fluka, $\geq 99.0 \%)$, 2-naphthoic acid (2-NpC, Sigma-Aldrich, $\geq 98 \%$ ), glycerol (spectrophotometric grade, $\geq 99.5 \%$, Sigma-Aldrich) and methanol (spectral grade, Fisher) were used as received. Sodium nitrite $\left(\mathrm{NaNO}_{2}, \mathrm{ACP}\right)$ was recrystallized from water once. Deionized water (Barnstead NANOpure deionizing systems $\geq 17.8 \mathrm{M} \Omega \mathrm{cm}$ ) was used for all sample preparations.

Sample Preparation: Appropriate amounts of F127 and water to prepare a $17 \%(w / w) ~ F 127$ solution were mixed in a sample vial and the contents were magnetically stirred in an ice bath until all the solid F127 was dissolved. The $17 \%(w / w)$ concentration of F127 corresponds to a $16 \mathrm{mM}$ monomer concentration of F127. This solution was left in the fridge overnight to remove any air bubbles formed during magnetic stirring. Water-glycerol mixtures, having the same viscosity of the aqueous $17 \%(\mathrm{w} / \mathrm{w}) \mathrm{F} 127$ solution at $20^{\circ} \mathrm{C}$, were prepared by mixing appropriate amounts of glycerol and water ${ }^{55}$ and were magnetically stirred for at least $3 \mathrm{~h}$ before the experiment. Stock solutions of $\mathrm{Np}(10 \mathrm{mM})$, Pht $(4 \mathrm{mM})$, 2- 
$\mathrm{NpOH}(10 \mathrm{mM})$ and $2-\mathrm{NpC}(10 \mathrm{mM})$ were prepared in methanol. A fresh stock solution of $\mathrm{NaNO}_{2}(1 \mathrm{M})$ was prepared in water on the day of the experiment. Appropriate amounts of the guest stock solution were added to the F127 solution or water-glycerol mixture to obtain a final concentration of $80 \mu \mathrm{M}$ each for $\mathrm{Np}, 2-\mathrm{NpOH}$ and 2-NpC. The concentrations of Pht in F127 solutions and water-glycerol mixtures were 50 and 30 $\mu \mathrm{M}$, respectively. The $\mathrm{pH}$ of the $\mathrm{F} 127$ solution with $2-\mathrm{NpC}$ was measured to be $6.5 \pm 0.1$ and the $\mathrm{pH}$ of the water used for preparing water-glycerol mixture with $2-\mathrm{NpC}$ was $6.2 \pm 0.2$. The required amount of $\mathrm{NaNO}_{2}$ was added to different $\mathrm{F} 127 /$ guest solutions to achieve the desired quencher concentration. After the addition of the guest and quencher, the samples were magnetically stirred in an ice bath for at least $10 \mathrm{~min}$ to ensure homogenization of the solution. This cold solution was then transferred to $7 \times 7 \mathrm{~mm}$ static Suprasil quartz cells used in the LFP measurements. During the experiment at $20^{\circ} \mathrm{C}$, the system was a sol and became a gel when the samples were heated to $30{ }^{\circ} \mathrm{C}$.

Instruments: The experiments were performed on a LFP system described elsewhere. ${ }^{56}$ The samples were excited at 266 nm using a Quanta Ray Lab-130 $4 \mathrm{~Hz}$ Nd:YAG laser from Spectra Physics. The sample temperature was controlled at 20 or $30{ }^{\circ} \mathrm{C}$ using a cryostat set-up from UNISOKU Scientific Instruments (USP-203). ${ }^{57}$ The samples were thermally equilibrated for at least $5 \mathrm{~min}$ before a measurement was made. The transient absorption was monitored at 415 or 420 $\mathrm{nm}$ for $\mathrm{Np}, 2-\mathrm{NpOH}$ and 2-NpC and at $490 \mathrm{~nm}$ for Pht, which correspond to the wavelengths where the maximum transient absorption was observed for these compounds.

\section{Results and discussion}

The addition of $\mathrm{NaNO}_{2}$ to $\mathrm{F} 127$ sol or gel containing the guests led to the quenching of the triplet excited states for all guests (Figures 1, S1-S5), where a faster decay of the triplet excited state leading to shorter excited state lifetimes and correspondingly higher observed decay rate constant $\left(k_{\text {obs }}\right)$ was observed. The decays for all guests and at all $\mathrm{NaNO}_{2}$ concentrations were adequately fit to a mono-exponential decay (see the Supporting Information for details on the fitting procedure), showing random residuals between the experimental data and the calculated fits (Figures S2-S5). The observation of mono-exponential decays supports the validity of the use of eqn 2 for the analysis of the quenching plots (see above).

The plots of $k_{o b s}$ with increasing quencher concentrations for each guest have a downward curvature (Figure 2). The curved quenching plots show that the guests are primarily located in the micelles, since a significant guest population in the aqueous phase of the gel or sol would have resulted in a linear quenching plot or the observation of a decay for the triplet states that is not mono-exponential. Out of the four guests
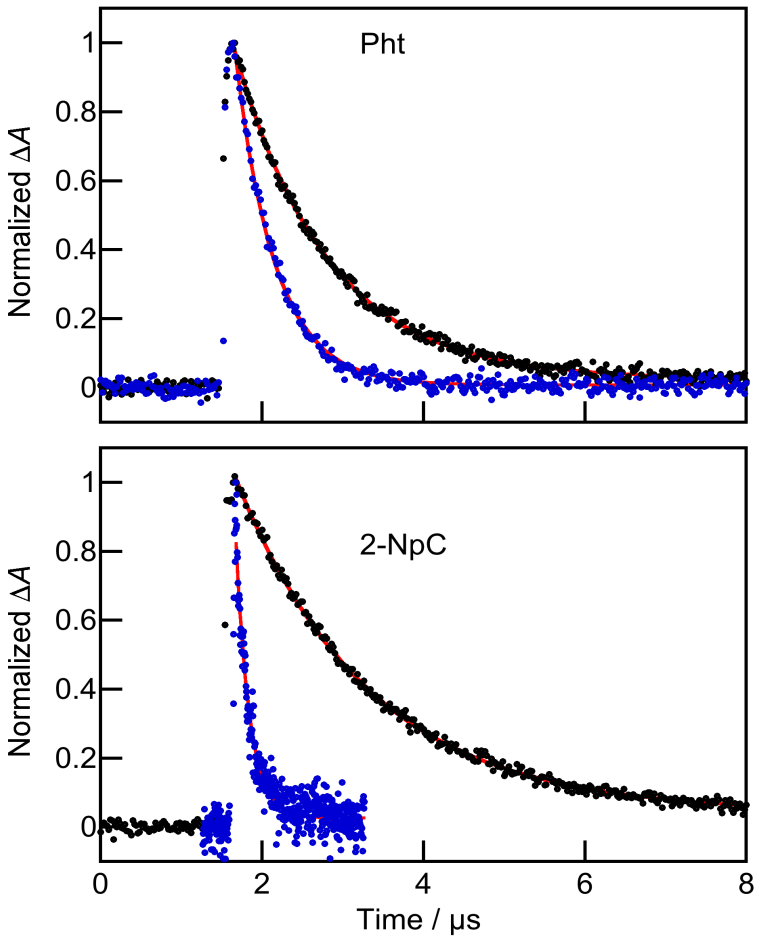

Figure 1. Normalized LFP decays for Pht (top) and 2-NpC (bottom) in the $\mathrm{F} 127 \mathrm{gel}$ in the absence (black) and presence (blue) of 12 $\mathrm{mM} \mathrm{NaNO}$. The red lines are the fits of the decays to a monoexponential function. The decay trace for $2-\mathrm{NpC}$ in the presence of nitromethane is offset on the abscissa to ensure that the time signifying the excitation is the same for both kinetic traces.

studied, 2-NpC is the least hydrophobic and has the highest probability of being located in the aqueous phase of the gel or sol. However, simulations of quenching plots for 2-NpC located in the aqueous phase (Figure S6) prove that this guest indeed is located in the micelles (see details in the Supporting Information). Qualitatively, the quenching is less efficient for Pht and $\mathrm{Np}$, followed by $2-\mathrm{NpOH}$ and $2-\mathrm{NpC}$, a trend that follows the hydrophobicities of the guests. This observation is to be expected because Pht and Np, being more hydrophobic, reside in the micellar core, thus making it difficult for the quencher to access them. On the other hand, 2- $\mathrm{NpOH}$ and 2$\mathrm{NpC}$, being less hydrophobic, reside in the corona region, where the quencher can access them more easily compared to guests bound to the micellar core.

For the analysis of the quenching plots using eqn 2 , several of the parameters $\left(N, k_{0}, k_{\mathrm{q}}, k_{0}^{F 127}\right.$ and $\left.k_{\mathrm{q}}^{F 127}\right)$ were fixed because they can be determined independently (Table 1). The aggregation number $(N)$ of 50 was assumed for $\mathrm{F} 127$ at both temperatures, because $N$ was shown to be constant with the micelle concentration and temperature. ${ }^{58}$ The value for $k_{0}^{F 127}$ was determined from the triplet excited state decay of the guest in $\mathrm{F} 127$ in the absence of any quencher. The $k_{\mathrm{q}}^{F 127}$ value was determined from the quenching plot at high quencher 


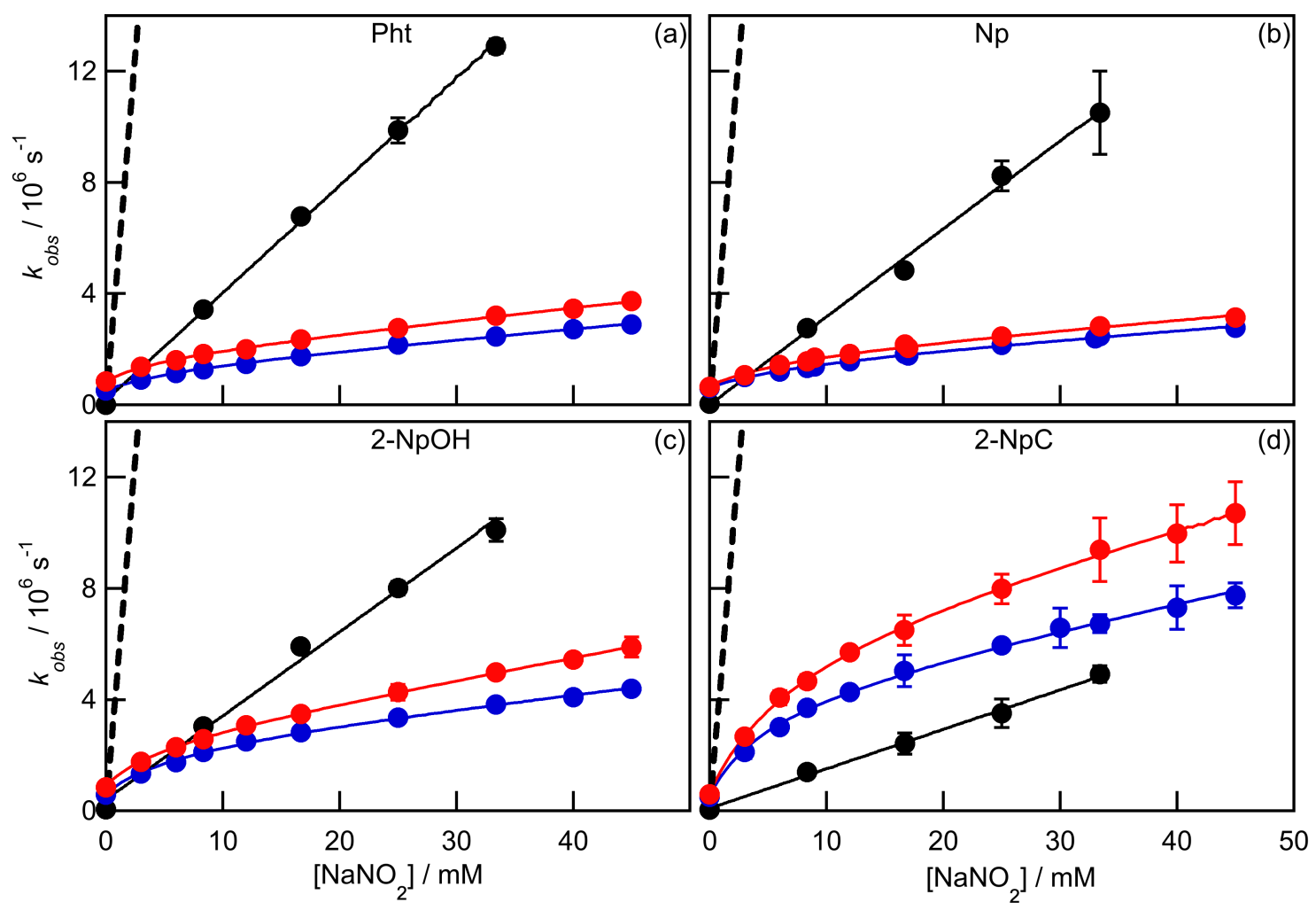

Figure 2. Quenching plots for the excited guests in water-glycerol (black), $17 \%$ (w/w) F127 at 20 (blue, sol) and $30{ }^{\circ} \mathrm{C}$ (red, gel). The scales for the abscissa and ordinates are the same to enable the comparison of the quenching plots. The dotted line represents the quenching of the guests by assuming a $k_{\mathrm{q}}$ value of $5 \times 10^{9} \mathrm{M}^{-1} \mathrm{~s}^{-1}$.

concentrations when the dependence of $k_{\text {obs }}$ on the quencher concentration is linear. The $k_{0}$ and $k_{\mathrm{q}}$ values were measured in a water-glycerol mixture that has the same macroscopic viscosity 55 as the $17 \%(\mathrm{w} / \mathrm{w}) \mathrm{F} 127$ sol at $20{ }^{\circ} \mathrm{C}$. However, determining the $k_{\mathrm{q}}$ value in the $\mathrm{F} 127 \mathrm{sol}$ and gel is not straightforward because F127, along with other Pluronics, are reported to have regions with much lower microviscosities, closer to that of aqueous solutions, compared to the macroscopic viscosity of the gel. ${ }^{18,23,37,38}$ Moreover, a gradient in microviscosity is observed in Pluronic micellar systems with the microviscosity increasing from the water-rich PEO corona towards the non-polar and dehydrated PPO core. ${ }^{23,36,38,39}$ Therefore, the upper and lower limits for $k_{\mathrm{q}}$ were defined respectively by the values measured in water-glycerol mixtures and a rate constant of $5 \times 10^{9} \mathrm{M}^{-1} \mathrm{~s}^{-1}$, which is close to the diffusion limit. ${ }^{59}$ In the case of 2-NpC, the quenching plot in the presence of $\mathrm{F} 127$ is above the quenching plot in water-glycerol, indicating that quenching rate constant in the aqueous phase of F127 is much higher than in the water-glycerol mixture. As a result, the water-glycerol mixture cannot be treated as an adequate solvent to estimate the minimum $k_{\mathrm{q}}$ for this guest. Studies with previous supramolecular systems, for which the $k_{\mathrm{q}}$ value is known, showed that the initial slope for the curved quenching plot is close to the slope for the quenching in homogeneous solution. ${ }^{45}$ Therefore, the minimum $k_{\mathrm{q}}$ value for 2-NpC was estimated from the initial slope of the quenching plots (Table 1).

The F127 micelles protect all excited guests from quenching by nitrite anions. The PE values are above 1 and range from 4 to 11 for different guests, indicating that all guests are located in the micelles, including 2-NpC (Table 1). The PE values for Pht and $\mathrm{Np}$ are higher compared to 2- $\mathrm{NpOH}$ and 2- $\mathrm{NpC}$, indicating that $\mathrm{Pht}$ and $\mathrm{Np}$ are located in a more protected environment. This result supports the assignment that $\mathrm{Pht}$ and $\mathrm{Np}$ are bound to the micellar core. The PE values for Pht and $\mathrm{Np}$ are the same and do not depend on the temperature, suggesting that $\mathrm{Np}$ and Pht are located in similar environments and that the accessibility of the quencher to the micellar core does not change when the micelles are immobilized in the gel compared to the sol. The PE values for 2- $\mathrm{NpOH}$ and $2-\mathrm{NpC}$ are the same in the sol phase at $20^{\circ} \mathrm{C}$. The PE for $2-\mathrm{NpOH}$ is lower in the gel, suggesting that the immobilization of micelles and relative ordering of the corona facilitates the access of the quencher to this guest. This easier access of the quencher could be due to 
the guest's relocation within the micelle or due to a lower diffusion barrier for the quencher into the micelle. The PE for $2-\mathrm{NpC}$ was the same in the sol and gel, suggesting that the binding site for this guest in the micelle is different from the binding site for $2-\mathrm{NpOH}$.

Table 1. Parameters fixed in the fits of the quenching plots for different triple excited state guests by nitrite anions in $17 \%$ $(w / w) ~ F 127$ at 20 and $30^{\circ} \mathrm{C}$. The errors correspond to average deviations from two experiments or standard deviations for three or more experiments.

\begin{tabular}{|c|c|c|c|c|c|c|c|}
\hline Guest & $\begin{array}{c}{[\mathrm{F} 127] /} \\
(w / w)\end{array}$ & $\mathrm{T} /{ }^{\circ} \mathrm{C}$ & $k_{0}^{F 127} / 10^{5} \mathrm{~s}^{-1}$ & $k_{0} / 10^{4} \mathrm{~s}^{-1}$ & $k_{q} / 10^{9} \mathrm{M}^{-1} \mathrm{~s}^{-1} \mathrm{a}$ & $k_{q}^{F 127} / 10^{7} \mathrm{M}^{-1} \mathrm{~s}^{-1}$ & $\mathbf{P E}^{\mathrm{b}}$ \\
\hline & 0 & 20 & & $0.84 \pm 0.01$ & & & \\
\hline \multirow[t]{3}{*}{ Pht } & $17 \%$ & 20 & $5.2 \pm 0.3$ & & $0.39 \pm 0.01$ & $3.7 \pm 0.1$ & $11 \pm 1$ \\
\hline & $17 \%$ & 30 & $8.5 \pm 0.3$ & & & $4.5 \pm 0.5$ & $9 \pm 1$ \\
\hline & 0 & 20 & & $4.7 \pm 0.1$ & & & \\
\hline \multirow[t]{3}{*}{$\mathrm{Np}$} & $17 \%$ & 20 & $6.0 \pm 0.2$ & & $0.32 \pm 0.01$ & $3.1 \pm 0.3$ & $10 \pm 1$ \\
\hline & $17 \%$ & 30 & $6.4 \pm 0.3$ & & & $3.4 \pm 0.5$ & $9 \pm 1$ \\
\hline & 0 & 20 & & $5.0 \pm 0.1$ & & & \\
\hline \multirow[t]{3}{*}{$2-\mathrm{NpOH}$} & $17 \%$ & 20 & $5.7 \pm 0.4$ & & $0.30 \pm 0.02$ & $4.8 \pm 0.6$ & $6.3 \pm 0.9$ \\
\hline & $17 \%$ & 30 & $8.4 \pm 0.7$ & & & $7.8 \pm 0.6$ & $3.9 \pm 0.4$ \\
\hline & 0 & 20 & & $3.8 \pm 0.1$ & & & \\
\hline \multirow[t]{2}{*}{ 2-NpC } & $17 \%$ & 20 & $5.1 \pm 0.1$ & & $0.61 \pm 0.08^{c}$ & $8.8 \pm 0.1$ & $6.9 \pm 0.9$ \\
\hline & $17 \%$ & 30 & $5.9 \pm 0.1$ & & & $11 \pm 1$ & $5.6 \pm 0.9$ \\
\hline
\end{tabular}

The association $\left(k_{\mathrm{as}}\right)$ and dissociation rate constants $\left(k_{\mathrm{dis}}\right)$ of the guests with the micelles were determined by fitting the quenching plots to eqn 2 assuming the minimum and maximum $k_{\mathrm{q}}$ values (Table 2). The values for $k_{\text {dis }}$ do not depend on the magnitude of $k_{\mathrm{q}}$, as expected for a unimolecular process. The values of $k_{\text {as }}$ depend on the magnitude of $k_{\mathrm{q}}$. The trend for the $k_{\mathrm{as}}$ values is the same for the analysis using the minimum or maximum $k_{\mathrm{q}}$ values. The $k_{\text {as }}$ values assuming the maximum $k_{\mathrm{q}}$ value are higher than the diffusion-controlled limit ${ }^{59}$ by at least one order of magnitude indicating that the $k_{\mathrm{q}}$ value is closer to the value determined in water-glycerol for these guests. For all guests, the $k_{\mathrm{as}}$ values determined for the minimum $k_{\mathrm{q}}$ values are within a factor of two to three of the diffusion-controlled rate constants in water $\left(6.5 \times 10^{9} \mathrm{M}^{-1} \mathrm{~s}^{-1}\right.$ and $7.4 \times 10^{9} \mathrm{M}^{-1} \mathrm{~s}^{-1}$ at 20 ${ }^{\circ} \mathrm{C}$ and $25^{\circ} \mathrm{C}$, respectively $\left.y^{59}\right)$, suggesting that the structure of the guest does not significantly affect the rate constant for association of the guests with the micelles.

Table 2. Dissociation $\left(k_{\text {dis }}\right)$ and association $\left(k_{\text {as }}\right)$ rate constants obtained from the fits of the quenching plots for the triplet excited state guests in $17 \%(\mathrm{w} / \mathrm{w}) \mathrm{F} 127$ at 20 and $30^{\circ} \mathrm{C}$.

\begin{tabular}{|c|c|c|c|c|c|}
\hline \multirow[t]{2}{*}{ Guest } & \multirow[t]{2}{*}{$\mathrm{T} /{ }^{\circ} \mathrm{C}$} & \multicolumn{2}{|c|}{ Minimum $k_{q}{ }^{b}$} & \multicolumn{2}{|c|}{ Maximum $\boldsymbol{k}_{q}{ }^{\mathrm{c}}$} \\
\hline & & $k_{\mathrm{dis}} / 10^{6} \mathrm{~s}^{-1}$ & $k_{a s} / 10^{9} \mathrm{M}^{-1} \mathrm{~s}^{-1}$ & $k_{\mathrm{dis}} / 10^{6} \mathrm{~s}^{-1}$ & $k_{a s} / 10^{9} \mathrm{M}^{-1} \mathrm{~s}^{-1}$ \\
\hline \multirow[t]{2}{*}{ Pht } & 20 & $0.85 \pm 0.03$ & $8 \pm 1$ & $0.85 \pm 0.03$ & $100 \pm 10$ \\
\hline & 30 & $0.97 \pm 0.03$ & $6.5 \pm 0.8$ & $0.97 \pm 0.03$ & $80 \pm 10$ \\
\hline \multirow[t]{2}{*}{$\mathrm{Np}$} & 20 & $0.99 \pm 0.04$ & $8 \pm 1$ & $0.98 \pm 0.04$ & $120 \pm 20$ \\
\hline & 30 & $1.2 \pm 0.1$ & $7 \pm 1$ & $1.2 \pm 0.1$ & $100 \pm 20$ \\
\hline \multirow[t]{2}{*}{$2-\mathrm{NpOH}$} & 20 & $2.0 \pm 0.1$ & $6.0 \pm 0.6$ & $1.9 \pm 0.1$ & $95 \pm 9$ \\
\hline & 30 & $1.7 \pm 0.1$ & $4.4 \pm 0.4$ & $1.7 \pm 0.1$ & $70 \pm 5$ \\
\hline \multirow[t]{2}{*}{$2-\mathrm{NpC}$} & 20 & $3.8 \pm 0.1$ & $10 \pm 1$ & $3.8 \pm 0.1$ & $79 \pm 8$ \\
\hline & 30 & $6.1 \pm 0.1$ & $14 \pm 1$ & $6.1 \pm 0.1$ & $116 \pm 7$ \\
\hline
\end{tabular}

[a], see the Supporting Information for individual $k_{\text {obs }}$ values at each quencher concentration (Tables S2-S5). ${ }^{[b]}, k_{\mathrm{q}}$ determined in water-glycerol mixtures with the same viscosity as $17 \% \mathrm{w} / \mathrm{w}$ F127 with the exception of $2-\mathrm{NpC}$. [c], $k_{\mathrm{q}}=5 \times 10^{9} \mathrm{M}^{-1} \mathrm{~s}^{-1}$. 
The release of guests from the micellar binding site, indicated by the $k_{\text {dis }}$ value, was found to be dependent on the guest's hydrophobicity (Table 2 ). The lowest $k_{\text {dis }}$ values were observed for Pht and $\mathrm{Np}$ followed by $2-\mathrm{NpOH}$ and then 2-NpC. The trend in $k_{\text {dis }}$ can be understood by the location of these guests in the micelles. In gels, Pht and Np, located in the micellar core, are released from the micelle with rate constants of $0.97 \times 10^{6} \mathrm{~s}^{-1}$ and $1.2 \times 10^{6} \mathrm{~s}^{-1}$, which correspond to residence lifetimes of 1.0 and $0.83 \mu \mathrm{s}$, respectively. The dissociation rate constant for $\mathrm{Np}$ is slightly higher than for Pht in line with the higher water solubility of Np. ${ }^{60}$ The $k_{\text {dis }}$ values for Pht and Np from micelles in the sol are similar to those in the gel, suggesting that the location of these guests in the core of the micelles does not change significantly when the system transitions from a sol to a gel.

In the case of 2-NpOH and 2- $\mathrm{NpC}$, a more nuanced picture emerges for the binding dynamics of these guests with the F127 micellar corona. The $k_{\text {dis }}$ value in the gel for $2-\mathrm{NpOH}$ of 1.7 $\times 10^{6} \mathrm{~s}^{-1}$ is higher than that for Pht and $\mathrm{Np}$, but significantly lower compared to $2-\mathrm{NpC}\left(6.1 \times 10^{6} \mathrm{~s}^{-1}\right)$. The different $k_{\text {dis }}$ values for $2-\mathrm{NpOH}$ and $2-\mathrm{NpC}$ supports the assignment made above that these two guests are located in different environments of the micellar corona. Previous studies with guests with different hydrophobicities showed that guests located in the corona can have different rotationalcorrelation $^{23,}{ }^{36-38}$ and translational-diffusion times. ${ }^{18}$ The guests rotate and diffuse faster when located closer to the peripheral region in the micellar corona. Thus, the higher $k_{\text {dis }}$ values for $2-\mathrm{NpC}$ is consistent with this guest being located closer to the periphery of the micelle, while $2-\mathrm{NpOH}$ is located closer to the PPO/PEO interface. The $k_{\text {dis }}$ values for $2-\mathrm{NpOH}$ are similar in the sol and gel. However, for $2-\mathrm{NpC}, k_{\text {dis }}$ in the gel is higher compared to the sol phase, showing that ordering of the corona in the gel facilitates the movement of small molecules bound to the periphery of the corona, but does not affect the dynamics of guests located in the micellar core or in the corona but closer to the PPO/PEO interface.

Pluronic micelles are more heterogeneous than conventional micelles, such as sodium dodecyl sulfate (SDS), making possible the control of the release kinetics from Pluronic micelles. Fluorescence studies of coumarin dyes C102 and C153, which have different hydrophobicities, were used to characterize SDS micelles ${ }^{61}$ and F88 Pluronic micelles. ${ }^{23,37}$ The qualitative trends for these dyes in SDS were the same, while a range of behaviours was observed in F88 because of the larger heterogeneity of Pluronic micelles. The dissociation rate constants of guests from micelles measured using the triplet excited state methodology also uncovered the presence of multiple binding sites in the hierarchical self-assembly of bile salt micelles when compared to SDS micelles. ${ }^{47,49,62}$ In the case of SDS micelles, ${ }^{47}$ the binding of guests is directly related to the guest's hydrophobicity indicating that SDS micelles provide an average hydrophobic environment. In contrast, in the bile salt self-assembled systems the presence of two binding sites with different hydrophobiciites were observed and the release kinetics of guests depend on the guest's hydrophobicity, size and shape with the dissociation rate constants varying by two orders of magnitude. ${ }^{49,62}$ F127 micelles are akin to bile salt selfassemblies with respect to the presence of multiple binding sites for guests. Therefore, structural modifications to the guest or the Pluronic micelles can affect the binding location of guests and consequently control the release time of guests from the micelles.

The determination of the guest-micelle dynamics at the molecular length scale provides a road map on how to alter the functionality of gels where transport of molecules and release of molecules from the gel material is desired. Our studies were performed at low loadings of guests to avoid changes to the micellar structure. ${ }^{63}$ The release kinetics of the guest from the micelles into the aqueous phase of the gel are not correlated with the gel's macroscopic viscosity, since similar values for $k_{\text {dis }}$ for Pht, $\mathrm{Np}$ and $2-\mathrm{NpOH}$ were observed in the sol and gel phases. A much lower value for $k_{\text {dis }}$ would be expected in the gel if these dynamics was affected by the gel's macroviscosity. This observation is in line with previous studies. For example, for F88 a Pluronic with a similar molecular weight to F127 but a slightly smaller core, the viscosity sensed by a guest in the micellar core is higher than in the corona. However, the microviscosity in the core is ca. $5 \times 10^{4}$ lower than the macroviscosity of the gel. ${ }^{23}$ Thus, the entanglement of the PEO chains in the gel does not affect, at the molecular scale, the potential energy surface for the exit of molecules from the micelles.

The dependence of the guest's hydrophobicity on the dynamics with F127 micelles in the sol and gel phases (Table 2) is in line with studies on the overall release of small molecules with different hydrophobicities from F127 gels into a surrounding medium. ${ }^{64}$ This overall release from the gel depends on the rates of the various elementary processes for the guest's dynamics inside the gel. The triplet excited state quenching experiments provide information at a molecular scale due to the finite lifetimes of these excited states. The guest's association rate constants are close to the diffusioncontrolled limit and the guest's hydrophobicity will not have a significant influence on its association rate. The diffusion of guests through the gel's "water" phase will be faster than dissociation of guests from the micelles, since "water" diffusion is less impeded than the guest's micellar exit. Therefore, the overall release of small molecules from the gel will be determined by the molecule's dissociation rate constant from the micelles. Based on this mechanistic understanding, the overall release kinetics for a molecule from the gel can be changed by modifying the micellar dissociation rate constant. A direct relationship between hydrophobicity and release kinetics will exist for molecules that reside in the micellar core, such as hydrophobic polyaromatic 
hydrocarbons. However, molecules that contain heteroatoms or hydrophilic groups, as for example most drugs do, will reside in the corona and a large change on the release kinetics can be achieved by changing the guest's structure to ensure that a molecule changes its location between the periphery and the interior of the corona. Such a relocation might also be achieved by changing the length of the PEO blocks and the PPO/PEO ratios.

\section{Conclusions}

The rational design of gels requires a mechanistic understanding to achieve the desired function for the material. In the case of dynamic functionalities, this understanding is achieved by studying the kinetics in real time. Triplet excited state quenching studies for the guests bound to different regions of F127 micelles in the gel and sol provided information at the molecular length scale on the guest binding dynamics to different sites in F127 micelles. Binding to the hydrophobic PPO micellar core led to the slowest release times, followed by the release time for a guest in the corona and close to the core, while the fastest release was observed for a guest at the periphery of the micelle. The guest-micelle binding dynamics was the same in the sol and gel for guests located in the core or in the interior of the corona, showing that studies for micelles in solution are predictive of the behavior in gels. For a guest bound to the periphery of the micelles a faster dynamics was observed in the gel when compared to the dynamics in the sol, suggesting that the entanglements of the PEO chains in the gel increase the dissociation of guests from the micelles. This nuanced picture for the guest binding dynamics shows that studies at the molecular length scale provide additional and complementary information to that obtained from techniques that probe the local mobility or the movement over several environments. These results provide the required information to tune the mobility of small molecules in gels and ultimately affect the overall release kinetics from these viscoelastic materials.

\section{Electronic Supporting Information}

Poisson distribution of guests in the micelles, fitting procedures, kinetics in the absence and presence of quencher, simulation of quenching plots, $k_{\text {obs }}$ values at individual quencher concentrations and rate constants fixed for the analysis of quenching plots.

\section{Author contributions}

Suma S. Thomas: Conceptualization, methodology development, initial investigation, supervision, final data analysis, writing.

Helia Hosseini-Nejad: Investigation, data analysis.
Cornelia Bohne: Conceptualization, supervision, writing, review and editing.

\section{Acknowledgements}

We acknowledge the Natural Sciences and Engineering Research Council of Canada (CREATE 497311-2017, RGPIN-2017-04458) for the partial support of this research and the donors of the American Chemical Society Petroleum Research Fund (58467-ND4) for the partial support of this research.

\section{Conflicts of Interest}

There are no conflicts to declare.

\section{References}

1. A. C. Barbati, J. Desroches, A. Robinsson and G. H. McKinley, Ann. Rev. Chem. Biomol. Eng., 2016, 7, 415-453.

2. L. Chen, J. Wang, L. Yu, Q. Zhang, M. Y. Fu, Z. Zhao and J. Zuo, Energy Fuels, 2018, 32, 6650-6656.

3. Y. Bai, S. He, T. Yu, C. Dai, J. Zhao and H. Zhang, ACS Omega, 2020, 5, 7244-7253.

4. H. Ullah, H. A. Santos and T. Khan, Cellulose, 2016, 23, 2291-2314.

5. S. Mitura, A. Sionkowska and A. Jaiswal, J. Mater. Sci. Mater. Med., 2020, 31, 50.

6. S. Cascone and G. Lamberti, Int. J. Pharm., 2020, 573, 118803.

7. H. Abdeltawab, D. Svirskis and M. Sharma, Expert Opin. Drug Deliv., 2020, 17, 495-509.

8. R. Dong, Y. Pang, Y. Su and X. Zhu, Biomater. Sci., 2015, 3, 937-954.

9. J. Li and D. J. Mooney, Nat. Rev. Mater., 2016, 1, 16071.

10. P. Majumder, U. Baxa, S. T. R. Walsh and J. P. Schneider, Angew. Chem. Int. Ed., 2018, 57, 15040-15044.

11. W. Xu, Q. Song, J.-F. Xu, M. J. Serpe and X. Zhang, ACS Appl. Mater. Interfaces, 2017, 9, 11368-11372.

12.S. Kwak and M. Lafleur, Macromolecules, 2003, 36, 31893195.

13. M. Shaikhullina, A. Khaliullina, R. Gimatdinov, A. Butakov, V. Chernov and A. Filippov, J. Mol. Liq., 2020, 306, 112898.

14. H. Walderhaug and B. Nyström, J. Phys. Chem. B, 1997, 101, 1524-1528.

15. F. Alvarez-Manceñido, K. Braechmans, S. C. De Smedt, J. Demeester, M. Landin and R. Martínez-Pacheco, Int. J. Pharm., 2006, 316, 37-46.

16. A. Berts, J.-E. Ehlers, S. Wöhl-Bruhn, H. Bunjes, K.-H. Gericke and H. Menzel, Macromol. Biosci., 2013, 13, 215226.

17. E. Schuster, A.-M. Hermansson, C. Öhgren, M. Rudemo and N. Lorén, Biophys. J., 2014, 106, 253-262. 
18. S. Ghosh, U. Mandal, A. Adhikari and K. Bhattacharyya, Chem. Asian J., 2009, 4, 948-954.

19. A. Michelman-Ribeiro, H. Boukari, R. Nossal and F. Horkay, Macromolecules, 2004, 37, 10212-10214.

20. A. Nandy, S. Chakraborty, S. Nandi, K. Bhattacharyya and S. Mukherjee, J. Phys. Chem. B, 2019, 123, 3397-3408.

21.S. Chakraborty, S. Nandi, K. Bhattacharyya and S. Mukherjee, ChemPhysChem, 2020, 21, 406-414.

22. U. Pramanik, S. Chakrabarti, K. Bhattacharyya and S. Mukherjee, Chem. Phys. Letters, 2021, 762, 138105.

23. C. D. Grant, K. E. Steege, M. R. Bunagan and E. W. Castner Jr., J. Phys. Chem. B, 2005, 109, 22273-22284.

24. M. Montalti, L. S. Dolci, L. Prodi, N. Zaccheroni, M. C. A. Stuart, K. J. C. van Bommel and A. Friggeri, Langmuir, 2006, 22, 2299-2303.

25. T. Hattori, K. Ishii, T. Tominaga, Y. Osada and T. Tahara, Chem. Phys., 2013, 419, 172-177.

26. F. Agnely, A. Djedour, A. Bochot and J. L. Grossiord, J. Drug Delivery Sci. Technol., 2006, 16, 3-10.

27.P. Alexandridis, Curr. Opin. Colloid Interface Sci., 1996, 1, 490-501.

28. I. R. Schmolka, Am. Perfum. Cosmet., 1967, 82, 25-30.

29. S. Fusco, A. Borzacchiello and P. A. Netti, J. Bioact. Compat. Polym., 2006, 21, 149-164.

30. G. Grassi, R. Farra, P. Caliceti, G. Guarnieri, S. Salmaso, M. Carenza and M. Grassi, Am. J. Drug Delivery, 2005, 3, 239251.

31. C. Yilin, A. Rodriguez, M. Vacanti, C. Ibarra, C. Arevalo and C. A. Vacanti, J. Biomater. Sci., Polym. Ed., 1998, 9, 475487.

32.P. Alexandridis and A. T. Hatton, Colloids Surf., A, 1995, 96, 1-46.

33. G. Wanka, H. Hoffmann and W. Ulbricht, Colloid Polym. Sci., 1990, 268, 101-117.

34. M. Malmsten and B. Lindman, Macromolecules, 1992, 25, 5440-5445.

35. S. Liu and L. Li, ACS Appl. Mater. Interfaces, 2015, 7, 26882697.

36. S. Jeon, S. Granick, K.-K. Kwon and K. Char, J. Polym. Sci., Part B: Polym. Phys., 2002, 40, 2883-2888.

37. C. D. Grant, M. R. DeRitter, K. E. Steege, T. A. Fadeeva and E. W. Castner Jr., Langmuir, 2005, 21, 1745-1752.

38. U. Anand and S. Mukherjee, Langmuir, 2014, 30, 10121021.

39. Y. Shiraishi, T. Inoue and T. Hirai, Langmuir, 2010, 26, 17505-17512.

40. Y. N. Degtyarev and S. Schlick, Langmuir, 1999, 15, 50405047.
41. M. Almgren, J. Alsins and P. Bahadur, Langmuir, 1991, 7, 446-450.

42. C. F. de Freitas, D. S. Pellosi, B. M. Estevão, I. R. Calori, T. M. Tsubone, M. J. Politi, W. Caetano and N. Hioka, Photochem. Photobiol., 2016, 92, 790-799.

43. T. Nivaggioli, P. Alexandridis and T. A. Hatton, Langmuir, 1995, 11, 730-737.

44. CRC Handbook of Chemistry and Physics, CRC Press, New York, 84th edn., 2003.

45. C. Bohne, Langmuir, 2006, 22, 9100-9111.

46. C. Bohne, Chem. Soc. Rev., 2014, 43, 4037-4050.

47. M. Almgren, F. Grieser and J. K. Thomas, J. Am. Chem. Soc., 1979, 101, 279-291.

48. T. C. Barros, K. Stefaniak, J. F. Holzwarth and C. Bohne, J. Phys. Chem. A, 1998, 102, 5639-5651.

49. O. Rinco, M.-C. Nolet, R. Ovans and C. Bohne, Photochem. Photobiol. Sci., 2003, 2, 1140-1151.

50. N. J. Turro, T. Okubo and C.-J. Chung, J. Am. Chem. Soc., 1982, 104, 1789-1794.

51. N. J. Turro, V. Ramamurthy and J. C. Scaiano, Principles of Molecular Photochemistry:An Introduction, University Science Books, Sausalito, 2009.

52.J. R. Lakowicz, Principles of Fluorescence Spectroscopy, Springer, Singapore, 3rd. edn., 2006.

53. M. H. Kleinman and C. Bohne, in Molecular and Supramolecular Photochemistry, eds. V. Ramamurthy and K. S. Schanze, Marcel Dekker Inc., New York, 1997, vol. 1, pp. 391-466.

54. M. H. Gehlen and F. C. De Schryver, Chem. Rev., 1993, 93, 199-221.

55. N.-S. Cheng, Ind. Eng. Chem. Res., 2008, 47, 3285-3288.

56. Y. Liao and C. Bohne, J. Phys. Chem., 1996, 100, 734-743.

57. T. C. S. Pace and C. Bohne, Can. J. Chem., 2011, 89, 395401.

58. R. K. Prud'homme, G. Wu and D. K. Schneider, Lagmuir, 1996, 12, 4651-4659.

59. M. Montalti, A. Credi, L. Prodi and M. T. Gandolfi, Handbook of Photochemistry, CRC Press, Boca Raton, 3rd edn., 2006.

60. R. S. Pearlman, S. H. Yalkowsky and S. Banerjee, J. Phys. Chem. Ref. Data, 1984, 13, 555-562.

61. H. Shirota, Y. Tamoto and H. Segawa, J. Phys. Chem. A, 2004, 108, 3244-3252.

62. L. L. Amundson, R. Li and C. Bohne, Langmuir, 2008, 24, 8491-8500.

63. R. Basak and R. Bandyopadhyay, Langmuir, 2013, 29, 4350-4356.

64. J. C. Gilbert, J. Hadgraft, A. Bye and L. G. Brookes, Int. J. Pharm., 1986, 12, 223-228. 


\title{
Electronic Supplementary Information
}

\section{Dynamics of small molecules within the F127 PEO-PPO-PEO triblock copolymer gel and sol phases studied at the molecular scale}

\author{
Suma S. Thomas ${ }^{1,2}$, Helia Hosseini-Nejad ${ }^{1,2}$ and Cornelia Bohne ${ }^{1,2 *}$ \\ ${ }^{1}$ Department of Chemistry, University of Victoria, P.O. Box 1700 STN CSC, Victoria, BC V8W \\ 2Y2, Canada. \\ ${ }^{2}$ Centre for Advanced Materials and Related Technology (CAMTEC), University of \\ Victoria,3800 Finnerty Rd, Victoria, BC, V8P 5C2, Canada
}

\section{INDEX}

1. Poisson distribution calculation for the guests in the micelles........................................... 2

2. Fitting procedure for the transient absorption decays ................................................... 2

3. Decay traces, fits and residuals for guests in F127 in the absence and presence of quencher ... 4

4. Simulation of quenching plots for 2-NpC in F127 assuming that most excited states are

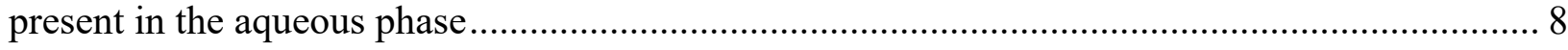

5. Values for $\mathrm{k}_{\mathrm{obs}}$ obtained from experiments ................................................................... 10

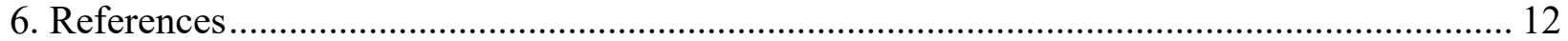




\section{Poisson distribution calculation for the guests in the micelles}

A Poisson distribution (eqn S1) was assumed for the distribution of guests in the F127 micelles. The fractions of micelles containing zero, one and more than one guest molecule were calculated. In eqn $\mathrm{S} 1, P$ corresponds to the fraction of micelles with $i$ number of guest molecules and $\bar{n}$ is the ratio between the guest to micelle concentrations. The monomer concentration of the F127 solution is $16 \mathrm{mM}$. The aggregation number $(N)$ of 50 was assumed for F127 micelles ${ }^{1}$ at both 20 and $30{ }^{\circ} \mathrm{C}$ to calculate the concentration of micelles. Of the occupied micelles, the maximum percentage of micelles containing two or more guests is calculated to be $12 \%$.

$$
P=\frac{\bar{n}^{i}}{i !} e^{-\bar{n}}
$$

Table S1. Poisson distribution for guests in F127 micelles assuming $N=50$.

Guests

Percentage of occupied micelles

$$
\text { with “ } i \text { ” number of guests }
$$

\begin{tabular}{ccccc}
\hline $\begin{array}{c}\text { Fraction of } \\
\text { micelles }\end{array}$ & $\begin{array}{c}\mathrm{Np}, 2-\mathrm{NpOH} \\
\text { and 2-NpC }\end{array}$ & $\mathrm{Pht}$ & $\begin{array}{c}\mathrm{Np}, 2-\mathrm{NpOH} \\
\text { and 2-NpC }\end{array}$ & Pht \\
\hline$P(i=0)$ & 0.7788 & 0.8553 & & \\
$P(i=1)$ & 0.1947 & 0.1336 & $88 \%$ & $92 \%$ \\
$P(i \geq 2)$ & 0.0265 & 0.0110 & $12 \%$ & $8 \%$ \\
\hline
\end{tabular}

\section{Fitting procedure for the transient absorption decays}

The transient absorption decays $(\Delta A)$ from the LFP experiments were fit to a monoexponential function (eqn $\mathrm{S} 2$ ), where $A_{0}$ and $A_{\infty}$ are the transient absorption values at the beginning and the end of the decay. The goodness of the fit was judged by the random distribution around zero of the residuals between the experimental data and the fit. Owing to the low signalto-noise ratio of LFP decays, good residuals were obtained for the same decay for a range of $A_{\infty}$ values, resulting in a range of acceptable value for $k_{o b s}$. Data fitting was done where the $A_{\infty}$ value for each fit was varied systematically from the lowest to the highest value for which good fits were obtained and the range of $k_{o b s}$ values determined were averaged. The standard deviation for the 
averaged values corresponds to the error associated with the averaged $k_{\text {obs }}$ value. For example, in Figure S1, the lifetime values of 733, 711 and 690 ns yielded fits with good residuals, leading to a value for $k_{o b s}$ of $(1.41 \pm 0.04) \times 10^{6} \mathrm{~s}^{-1}$. The fits with lifetime values of 767 and $661 \mathrm{~ns}$ led to nonrandom residuals around zero, and therefore were not considered as good fits.

$\Delta A=A_{0} e^{-k_{o b s} t}+A_{\infty}$

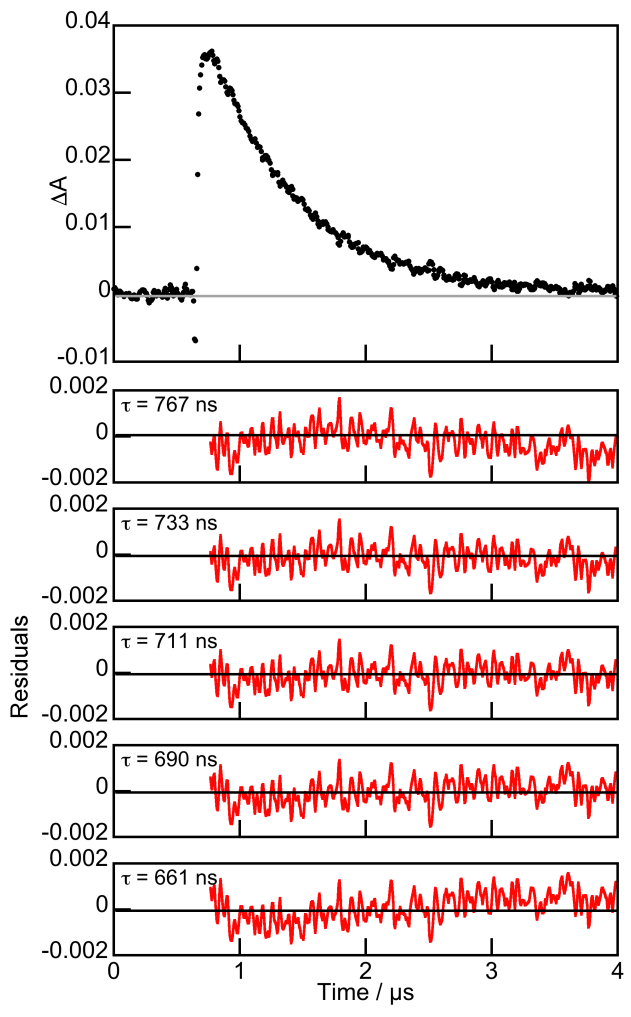

Figure S1. LFP decay for $50 \mu \mathrm{M}$ Pht in $17 \%(w / w) ~ F 127$ at $30{ }^{\circ} \mathrm{C}$ in the presence of $3 \mathrm{mM}$ $\mathrm{NaNO}_{2}$ and the residuals between the experimental data and the calculated fit of the decay with different $A_{\infty}$ values, yielding lifetime values from $767 \mathrm{~ns}$ (top) to $661 \mathrm{~ns}$ (bottom). 


\section{Decay traces, fits and residuals for guests in F127 in the absence and presence of quencher}

The LFP decays of all the guests in 17\% (w/w) F127 were mono-exponential at both 20 and $30{ }^{\circ} \mathrm{C}$ in the absence and presence of quencher.
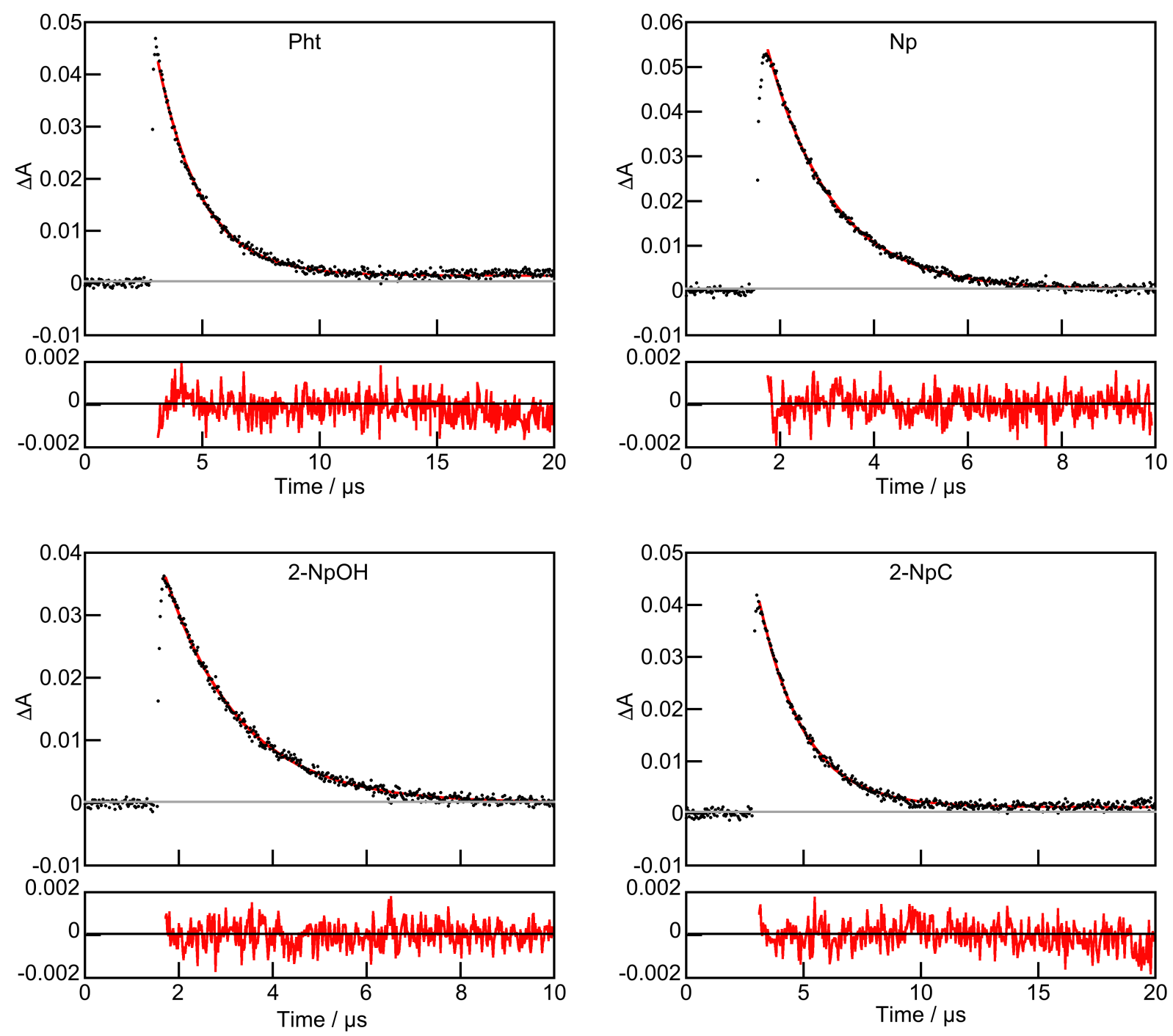

Figure S2. LFP decay traces, fits to a mono-exponential function and corresponding residuals between the experimental data and the calculated fit for the guests in $17 \%(\mathrm{w} / \mathrm{w}) \mathrm{F} 127$ at $20{ }^{\circ} \mathrm{C}$ in the absence of quencher. 

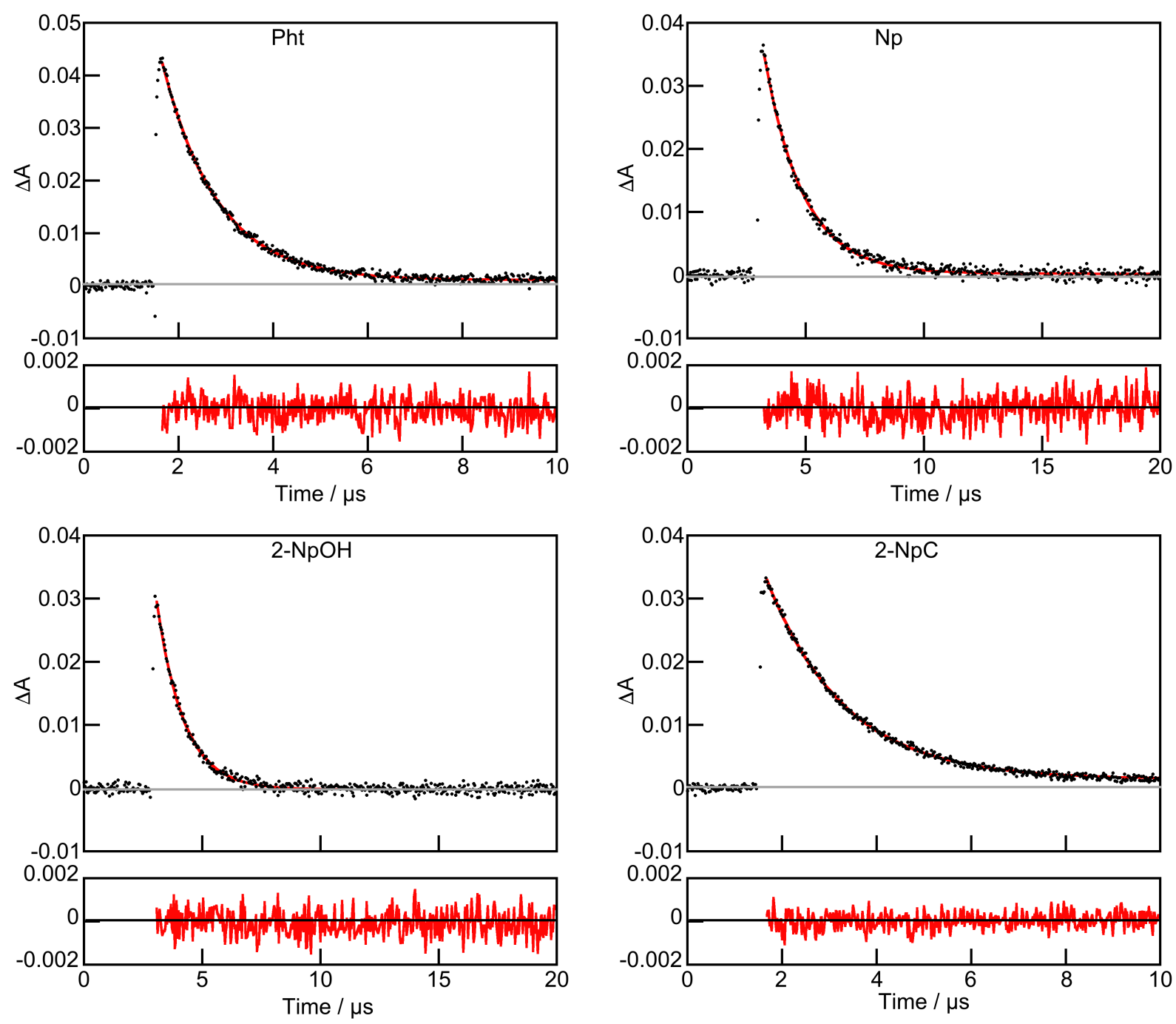

Figure S3. LFP decay traces, fits to a mono-exponential function and corresponding residuals between the experimental data and the calculated fit for the guests in $17 \%(\mathrm{w} / \mathrm{w}) \mathrm{F} 127$ at $30{ }^{\circ} \mathrm{C}$ in the absence of quencher. 

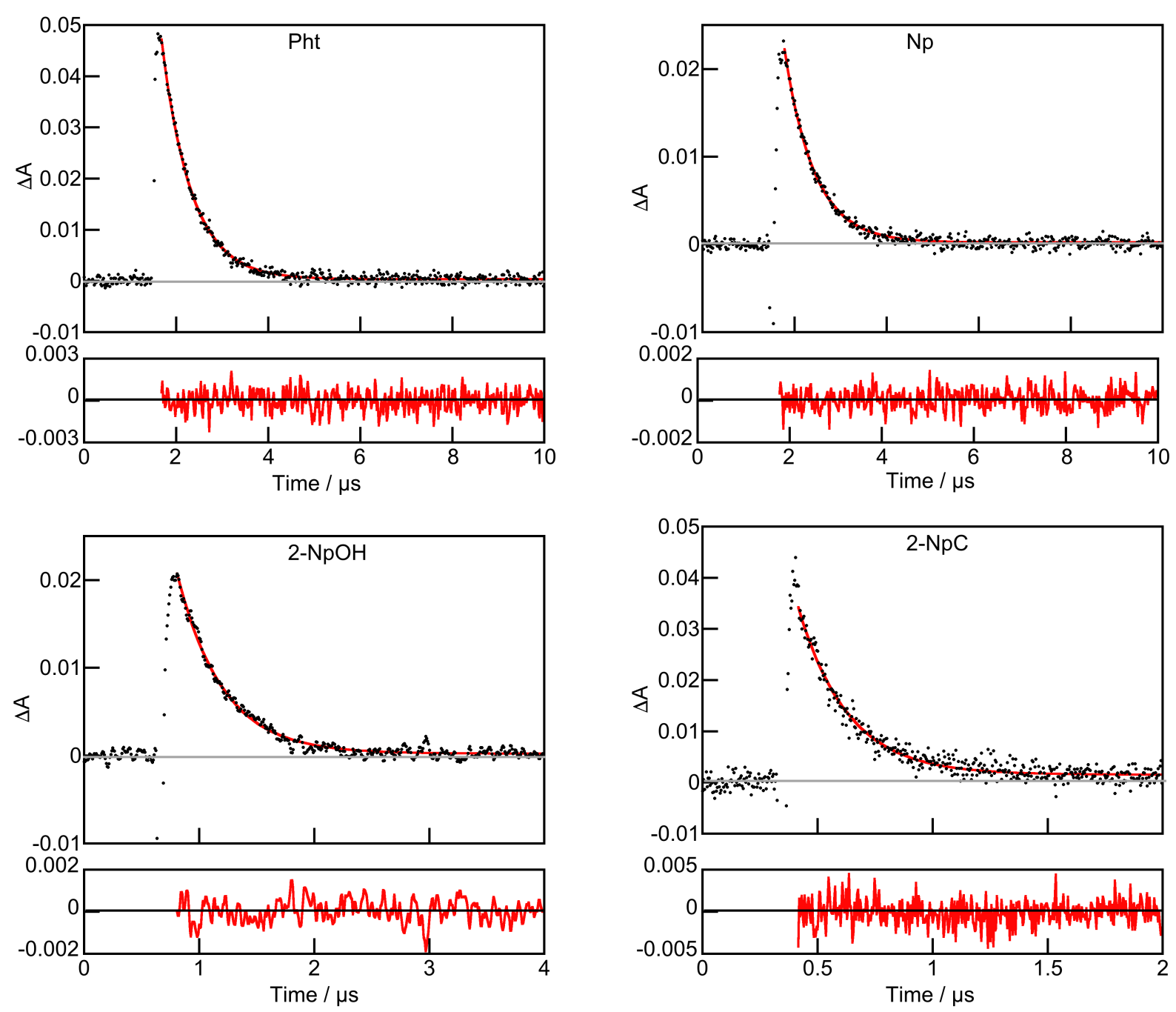

Figure S4. LFP decay traces, fits to a mono-exponential function and corresponding residuals between the experimental data and the calculated fit for the guests in $17 \%(\mathrm{w} / \mathrm{w}) \mathrm{F} 127$ at $20^{\circ} \mathrm{C}$ in the presence of $12 \mathrm{mM} \mathrm{NaNO}_{2}$. 

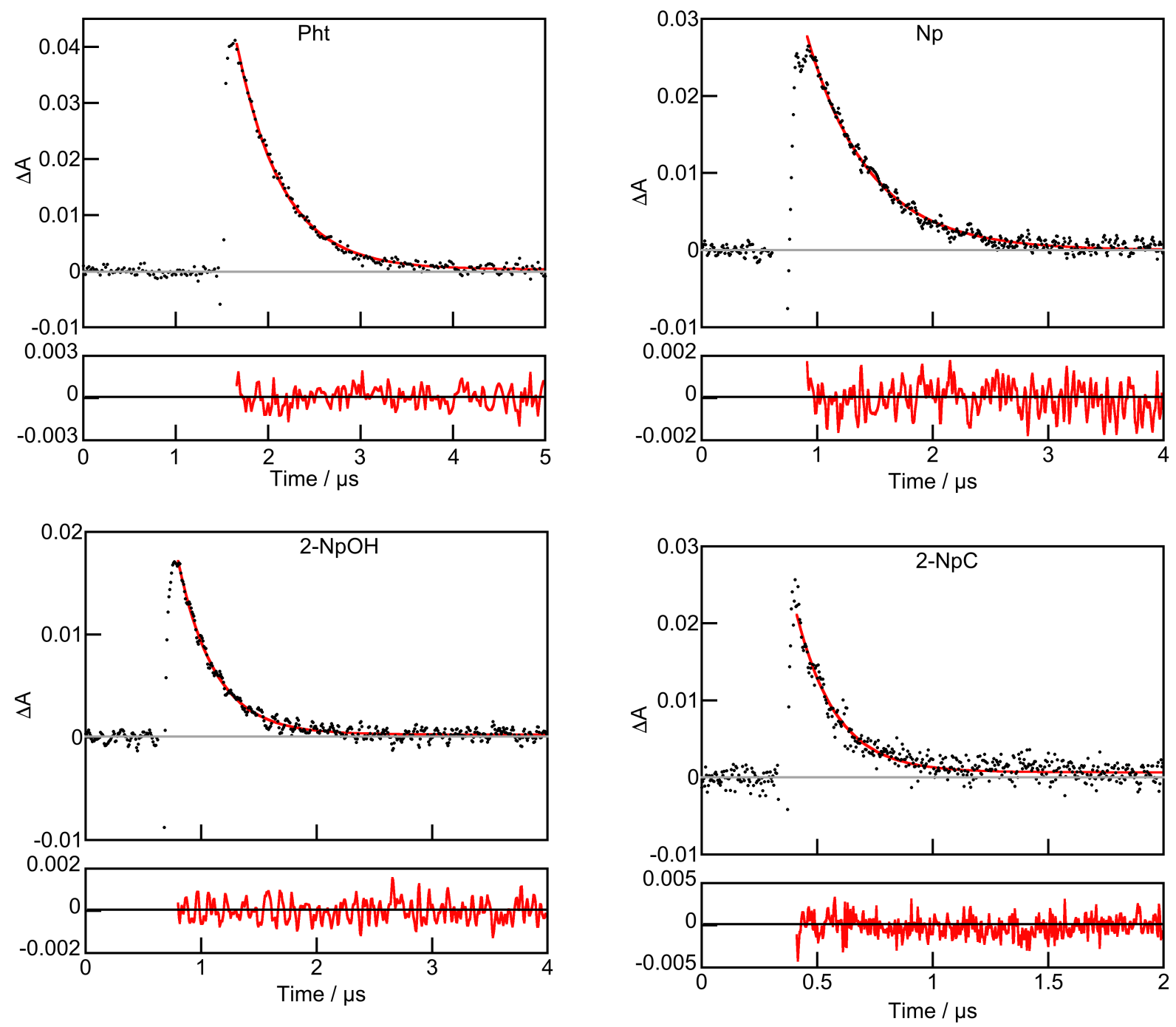

Figure S5. LFP decay traces, fits to a mono-exponential function and corresponding residuals between the experimental data and the calculated fit for the guests in $17 \%(\mathrm{w} / \mathrm{w}) \mathrm{F} 127$ at $30{ }^{\circ} \mathrm{C}$ in the presence of $12 \mathrm{mM} \mathrm{NaNO}_{2}$. 


\section{Simulation of quenching plots for $2-\mathrm{NpC}$ in F127 assuming that most excited states are present in the aqueous phase}

The reactions involved in Scheme 1 of the paper are given below. P, M and MP represents the guest, F127 micelle and the guest bound to the micelles, respectively. The excited state of the guest is represented by the asterisk.

$$
\begin{aligned}
& \mathrm{MP}^{*} \underset{k_{d i s}}{\stackrel{k_{a s}}{\longrightarrow}} \mathrm{M}+\mathrm{P}^{*} \\
& \mathrm{P}^{*} \stackrel{k_{0}}{\longrightarrow} \mathrm{P}+\mathrm{h} v \\
& \mathrm{MP}^{*} \stackrel{k_{0}^{F 127}}{\longrightarrow} \mathrm{MP}+\mathrm{h} v \\
& \mathrm{P}^{*}+\mathrm{Q} \stackrel{k_{q}}{\longrightarrow} \mathrm{P} \\
& \mathrm{MP}^{*}+\mathrm{Q} \stackrel{k_{q}^{F 127}}{\longrightarrow} \mathrm{MP}
\end{aligned}
$$

The rate laws for the concentrations of $\mathrm{MP}^{*}$ and $\mathrm{P}^{*}$ are given by eqns $\mathrm{S} 8$ and $\mathrm{S} 9$. The concentration of the micelle is calculated from the F127 monomer concentration and $N$.

$$
\begin{aligned}
& \frac{-d\left[M P^{*}\right]}{d t}=k_{d i s}\left[M P^{*}\right]-k_{a s}[M]\left[P^{*}\right]+k_{0}^{F 127}\left[M P^{*}\right]+k_{q}^{F 127}\left[M P^{*}\right][Q] \\
& \frac{-d\left[P^{*}\right]}{d t}=k_{a s}[M]\left[P^{*}\right]-k_{d i s}\left[M P^{*}\right]+k_{0}\left[P^{*}\right]+k_{q}\left[P^{*}\right][Q]
\end{aligned}
$$

If the amount of guest in water is much higher compared to that in the micelles, then steady state can be assumed for the concentration of the excited guests in the micelles, leading to the following equation.

$$
\frac{-d\left[M P^{*}\right]}{d t}=0
$$

From eqns S8 and S10 eqn S11 is derived.

$$
\left[M P^{*}\right]=\frac{k_{a s}[M]\left[P^{*}\right]}{k_{d i s}+k_{0}^{F 127}+k_{q}^{F 127}[Q]}
$$


Substituting for $\left[\mathrm{MP}^{*}\right]$ in eqn $\mathrm{S}$, the following equation is derived.

$\frac{-d\left[P^{*}\right]}{d t}=k_{a s}[M]\left[P^{*}\right]-\frac{k_{d i s} k_{a s}[M]\left[P^{*}\right]}{k_{d i s}+k_{0}^{F 127}+k_{q}^{F 127}[Q]}+k_{0}\left[P^{*}\right]+k_{q}\left[P^{*}\right][Q]$

Rearranging eqn S12, eqn S13 is obtained.

$\frac{-d\left[P^{*}\right]}{d t}=\left(k_{a s}[M]+k_{0}+k_{q}[Q]-\frac{k_{d i s} k_{a s}[M]}{k_{d i s}+k_{0}^{F 127}+k_{q}^{F 127}[Q]}\right)\left[P^{*}\right]$

Thus, the observed rate constant is given by eqn $\mathrm{S} 14$.

$k_{o b s}=k_{a s}[M]+k_{0}+k_{q}[Q]-\frac{k_{d i s} k_{a s}[M]}{k_{d i s}+k_{0}^{F 127}+k_{q}^{F 127}[Q]}$

In the set of guests we used, $2-\mathrm{NpC}$ is the most hydrophilic one. Therefore, this guest has the highest probability of being located mostly in the aqueous phase. Assuming the parameter values described in the paper, the quenching plot for 2-NpC in F127 was simulated using eqn S14 and was found to be linear for different values of $k_{a s}$ and $k_{q}$ (Figure S6). Thus, 2-NpC is confirmed to be located in the micelles because the experimentally observed quenching plot is curved.

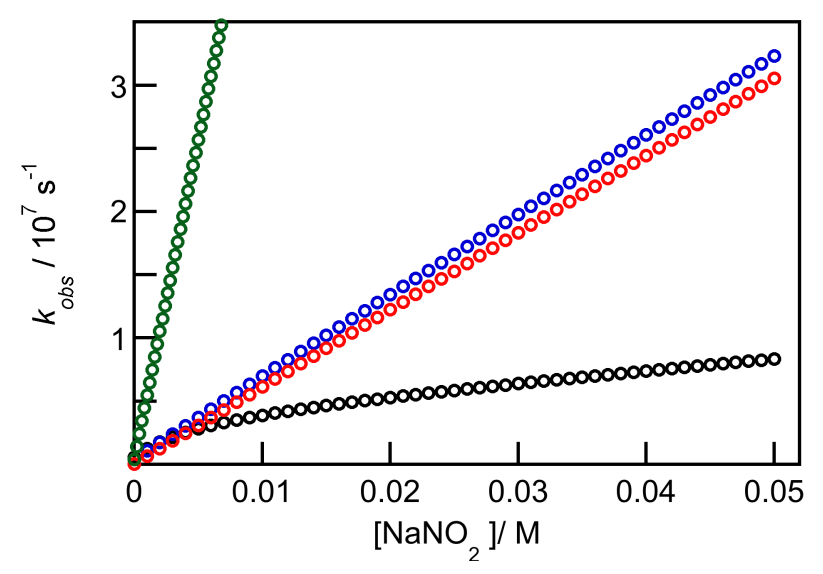

Figure S6. Simulated quenching plots for 2-NpC using eqn S14 assuming $k_{a s}$ values of $1 \times 10^{10}$ (blue), $1 \times 10^{7} \mathrm{M}^{-1} \mathrm{~s}^{-1}$ (red) and a $\mathrm{k}_{\mathrm{q}}$ value of $5 \times 10^{9} \mathrm{M}^{-1} \mathrm{~s}^{-1}$ (green). Except for the assumed values mentioned above, all other parameters were fixed to values for $2-\mathrm{NpC}$ in $\mathrm{F} 127$ at $20^{\circ} \mathrm{C}$ from Tables 1 and 2 . The black curve represents the simulated quenching plot for $2-\mathrm{NpC}$ using eqn 2 in the paper. 


\section{Values for $\boldsymbol{k}_{\text {obs }}$ obtained from experiments}

The $k_{o b s}$ values and corresponding errors used for the quenching plots are given in Tables $\mathrm{S} 2-\mathrm{S} 5$. The errors for the $k_{o b s}$ values were obtained from the average or standard deviations from the systematic fits explained in section 2 above when one independent quenching experiment was done. The average deviations or the error derived from the systematic fits, whichever is higher, is reported where two independent experiments were done, and standard deviations are reported where three independent experiments were performed. For all guests, the error values at higher quencher concentrations were larger compared to values at low quencher concentrations due to the lower signal-to-noise ratio for the decays at higher quencher concentrations. As the quencher concentration increases, the triplet excited state lifetimes become shorter and interference from fluorescence can occur. In addition, the initial amplitude of the transient absorption signal decreases because of quenching of the singlet excited state of the guests. In the case of 2-NpC, the errors for $k_{o b s}$ values at higher quencher concentrations are higher compared to other guests because of the distortion of the decay due to the interference of fluorescence signals from this guest at the monitoring wavelength.

Table S2. Values for $k_{o b s}$ for Pht in water-glycerol and F127 micelles at 20 and $30^{\circ} \mathrm{C}^{\mathrm{a}}$

\begin{tabular}{cccc}
\hline \multicolumn{4}{c}{$\boldsymbol{k}_{\mathbf{o b s}} / \mathbf{1 0}^{\mathbf{6}} \mathbf{s}^{\mathbf{- 1}}$} \\
\hline $\begin{array}{c}\left.\mathbf{N a N O}_{2}\right] / \\
\mathbf{m M}\end{array}$ & Water-glycerol & $\mathbf{F 1 2 7} / \mathbf{2 0}{ }^{\circ} \mathbf{C}$ & $\mathbf{F 1 2 7 / 3 0}{ }^{\circ} \mathbf{C}$ \\
\hline 0 & $0.00837 \pm 0.00001$ & $0.52 \pm 0.03(2)$ & $0.85 \pm 0.02(2)$ \\
3 & $(2)$ & $0.9113 \pm 0.0004(2)$ & $1.41 \pm 0.04(1)$ \\
6 & - & $1.15 \pm 0.02(1)$ & $1.60 \pm 0.04(1)$ \\
8.3 & - & $1.27 \pm 0.03(2)$ & $1.82 \pm 0.03(1)$ \\
12 & - & $1.47 \pm 0.03(2)$ & $2.00 \pm 0.03(1)$ \\
16.7 & $6.8 \pm 0.2(2)$ & $1.75 \pm 0.02(2)$ & $2.4 \pm 0.1(1)$ \\
25 & $10.0 \pm 0.5(2)$ & $2.17 \pm 0.03(2)$ & $2.76 \pm 0.06(2)$ \\
33.4 & $12.9 \pm 0.3(2)$ & $2.46 \pm 0.04(2)$ & $3.21 \pm 0.09(1)$ \\
40 & - & $2.7 \pm 0.1(1)$ & $3.45 \pm 0.05(1)$ \\
45 & - & $2.9 \pm 0.1(1)$ & $3.73 \pm 0.03(1)$ \\
\hline
\end{tabular}

a, number of independent experiments shown in parenthesis 
$\underline{\text { Table S3. Values for } k_{o b s} \text { for } \mathrm{Np} \text { in water-glycerol and F127 micelles at } 20 \text { and } 30^{\circ} \mathrm{C}}$

\begin{tabular}{cccc}
\hline \multicolumn{4}{c}{$\boldsymbol{k}_{\mathbf{0 b s}} / \mathbf{1 0}^{\mathbf{6}} \mathbf{s}^{-\mathbf{1}}$} \\
\hline $\begin{array}{c}\left.\mathbf{N a N O} \mathbf{O}_{2}\right] / \\
\mathbf{m M}\end{array}$ & Water-glycerol & $\mathbf{F 1 2 7} / \mathbf{2 0}{ }^{\circ} \mathbf{C}$ & $\mathbf{F 1 2 7 / 3 0}{ }^{\circ} \mathbf{C}$ \\
\hline 0 & $0.047 \pm 0.001(3)$ & $0.60 \pm 0.02(2)$ & $0.64 \pm 0.03(2)$ \\
3 & - & $1.0 \pm 0.1(1)$ & $1.07 \pm 0.04(1)$ \\
6 & - & $1.20 \pm 0.01(1)$ & $1.43 \pm 0.06(1)$ \\
8.3 & $2.8 \pm 0.2(3)$ & $1.332 \pm 0.007(2)$ & $1.56 \pm 0.02(2)$ \\
9 & - & $1.382 \pm 0.002(1)$ & $1.69 \pm 0.04(1)$ \\
12 & - & $1.557 \pm 0.009(1)$ & $1.82 \pm 0.02(1)$ \\
16.7 & $4.84 \pm 0.03(2)$ & $1.82 \pm 0.01(2)$ & $2.2 \pm 0.1(2)$ \\
17 & - & $1.78 \pm 0.06(1)$ & $2.05 \pm 0.06(1)$ \\
25 & $8.2 \pm 0.5(3)$ & $2.2 \pm 0.1(3)$ & $2.5 \pm 0.1(3)$ \\
33 & - & $2.39 \pm 0.05(1)$ & - \\
33.4 & $11 \pm 2(3)$ & $2.5 \pm 0.1(2)$ & $2.8 \pm 0.1(2)$ \\
45 & - & $2.77 \pm 0.02(1)$ & $3.1 \pm 0.2(1)$ \\
\hline
\end{tabular}

a, number of independent experiments shown in parenthesis

Table S4. Values for $k_{o b s}$ for 2-NpOH in water-glycerol and F127 micelles at 20 and $30^{\circ} \mathrm{C}$

\begin{tabular}{|c|c|c|c|}
\hline \multicolumn{4}{|c|}{$k_{\text {obs }} / 10^{6} \mathrm{~s}^{-1}$} \\
\hline $\begin{array}{c}{\left[\mathrm{NaNO}_{2}\right] /} \\
\mathbf{m M}\end{array}$ & Water-glycerol & F127 / $20^{\circ} \mathrm{C}$ & $\mathrm{F} 127 / 30^{\circ} \mathrm{C}$ \\
\hline 0 & $0.0500 \pm 0.0004(2)$ & $0.57 \pm 0.04(3)$ & $0.84 \pm 0.07(3)$ \\
\hline 3 & - & $1.34 \pm 0.02$ & $1.76 \pm 0.04(1)$ \\
\hline 6 & - & $1.75 \pm 0.04$ & $2.3 \pm 0.1$ \\
\hline 8.3 & $3.03 \pm 0.06(2)$ & $2.11 \pm 0.02(2)$ & $2.6 \pm 0.2(2)$ \\
\hline 12 & - & $2.50 \pm 0.05$ & $3.08 \pm 0.08(1)$ \\
\hline 16.7 & $5.9 \pm 0.1(2)$ & $2.8 \pm 0.1(2)$ & $3.5 \pm 0.2(2)$ \\
\hline 25 & $8.0 \pm 0.2(2)$ & $3.4 \pm 0.1(2)$ & $4.3 \pm 0.3(2)$ \\
\hline 33.4 & $10.1 \pm 0.4(2)$ & $3.8 \pm 0.1(1)$ & $4.984 \pm 0.004$ \\
\hline 40 & - & $4.09 \pm 0.06(1)$ & $5.4 \pm 0.2(1)$ \\
\hline 45 & - & $4.39 \pm 0.07(1)$ & $5.9 \pm 0.4(1)$ \\
\hline
\end{tabular}

a , number of independent experiments shown in parenthesis 
Table S5. Values for $k_{o b s}$ for 2-NpC in water-glycerol and F127 micelles at 20 and $30^{\circ} \mathrm{C}$

\begin{tabular}{cccc}
\hline \multicolumn{4}{c}{$\boldsymbol{k}_{\mathbf{o b s}} / \mathbf{1 0}^{\mathbf{6}} \mathbf{s}^{-\mathbf{1}}$} \\
\hline $\begin{array}{c}\text { [NaNO} \\
\mathbf{m M}\end{array}$ & Water-glycerol & $\mathbf{F 1 2 7} / \mathbf{~ 2 0}^{\circ} \mathbf{C}$ & $\mathbf{F 1 2 7 / 3 0}{ }^{\circ} \mathbf{C}$ \\
\hline 0 & $0.0383 \pm 0.0008(2)$ & $0.506 \pm 0.009(2)$ & $0.592 \pm 0.002(2)$ \\
3 & - & $2.1 \pm 0.2(1)$ & $2.66 \pm 0.06(1)$ \\
6 & - & $3.0 \pm 0.2(1)$ & $4.1 \pm 0.3(1)$ \\
8.3 & $1.403 \pm 0.004(2)$ & $3.714 \pm 0.008(1)$ & $4.7 \pm 0.2(1)$ \\
12 & - & $4.3 \pm 0.1(1)$ & $5.7 \pm 0.2(1)$ \\
16.7 & $2.4 \pm 0.4(2)$ & $5.0 \pm 0.6(1)$ & $6.5 \pm 0.5(1)$ \\
25 & $3.5 \pm 0.5(2)$ & $6.0 \pm 0.2(1)$ & $8.0 \pm 0.5(1)$ \\
30 & $4.9 \pm 0.3(2)$ & $6.6 \pm 0.7(1)$ & - \\
33.4 & - & $6.7 \pm 0.3(1)$ & $9 \pm 1(1)$ \\
40 & - & $7.3 \pm 0.8(1)$ & $10 \pm 1(1)$ \\
45 & & $7.8 \pm 0.5(1)$ & $11 \pm 1(1)$ \\
\hline
\end{tabular}

${ }^{\text {a }}$, number of independent experiments shown in parenthesis

\section{References}

(1) R. K. Prud'homme, G. Wu and D. K. Schneider, Lagmuir, 1996, 12, 4651-4659. 